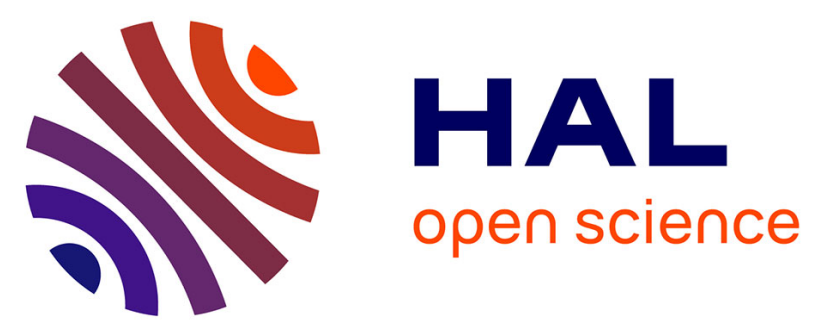

\title{
Chemical and growth traits of the peach tree may induce higher infestation rates of the green peach aphid, Myzus persicae (Sulzer)
}

\author{
Marie Odile Jordan, Marie-Helene Sauge, Gilles Vercambre
}

\section{To cite this version:}

Marie Odile Jordan, Marie-Helene Sauge, Gilles Vercambre. Chemical and growth traits of the peach tree may induce higher infestation rates of the green peach aphid, Myzus persicae (Sulzer). Pest Management Science, 2019, 76 (2), pp.797-806. 10.1002/ps.5583 . hal-02283440

HAL Id: hal-02283440

https://hal.science/hal-02283440

Submitted on 26 May 2020

HAL is a multi-disciplinary open access archive for the deposit and dissemination of scientific research documents, whether they are published or not. The documents may come from teaching and research institutions in France or abroad, or from public or private research centers.
L'archive ouverte pluridisciplinaire HAL, est destinée au dépôt et à la diffusion de documents scientifiques de niveau recherche, publiés ou non, émanant des établissements d'enseignement et de recherche français ou étrangers, des laboratoires publics ou privés.

\section{(1) (1) $\$$}

Distributed under a Creative Commons Attribution - NonCommercial - NoDerivatives $\mid 4.0$ 


\title{
Chemical and growth traits of the peach tree may induce higher infestation rates of the green peach aphid, Myzus persicae (Sulzer)
}

Marie-Odile Jordan $^{\mathbf{a}^{*}}$, Marie-Hélène Sauge ${ }^{\mathbf{a}}$, Gilles Vercambre ${ }^{\mathbf{a}}$

a INRA, UR1115 Plantes et Systèmes de Culture Horticoles (PSH), Domaine Saint-Paul, Site Agroparc, 84914 Avignon cedex 09, France

*corresponding author: Marie-Odile Jordan; email: marie-odile.jordan@inra.fr

\begin{abstract}
BACKGROUND: The green peach aphids, Myzus persicae, are a predominant pest in peach orchards as they can alter fruiting and shoot development for several years. This aphid developed resistance against pesticides. Among the alternative control strategies is the reduction of trees attractiveness to aphids. In order to identify the plant variables related to plant susceptibility, young peach trees were submitted to various supplies of water and nitrogen, and then artificially infested with aphids. Shoot development, plant water potential and aphid abundance were then monitored on a weekly basis. The apex concentrations in total $\mathrm{N}$, amino acids, soluble sugars and polyphenols were determined at infestation start and infestation peak.
\end{abstract}

This article has been accepted for publication and undergone full peer review but has not been through the copyediting, typesetting, pagination and proofreading process which may lead to differences between this version and the Version of Record. Please cite this article as doi: $10.1002 /$ ps.5583 
RESULTS: Until infestation peak, the thermal time requests for aphid development were independent of infestation severity. The aphid populations collapsed then more rapidly on the low infested shoots than on the high infested ones. Aphid abundances appeared to be positively related to shoot development (leaf expansion and secondary ramification), to shoot growth (stem length and diameter), and to apex concentrations in amino acids and nonstructural carbohydrates (NSC). Polyphenols had an opposite effect.

CONCLUSION: Peach susceptibility to aphids depends on shoot development and apex composition, and could be lowered by decreasing the water and nitrogen inputs.

Keywords: Bottom-up control, Myzus persicae, Prunus persica, shoot development, shoot composition, pest management

\section{Introduction}

Conventional agriculture relies on the use of a high level of chemical inputs such as fertilizers and pesticides. Increasing fruit production sustainability requires the development of alternatives to pesticides, insofar as orchards are the most intensively sprayed culture. In peach orchards (Prunus persica L. Batsch), among the numerous herbivorous pests, the green peach aphid (Myzus persicae Sulzer) is considered to be the most economically important one. ${ }^{1}$ This pest influences fruit production for several years, ${ }^{2}$ either directly by fruit deformation, fruit fall or decrease of fruit size and sugar content, ${ }^{3}$ or indirectly through its effects on shoot growth and ability to transmit plant viruses. ${ }^{4}$ However, the control of the green peach aphid through chemicals is being increasingly difficult due to its development of resistance to the most frequently used pesticides ${ }^{1}$ and to the implementation of restrictive 
guidelines.

Thus, alternative methods have to be mobilized. Among them, biological control methods aiming to regulate the aphid populations by predation or parasitism are well documented. ${ }^{5}$ They intend to foster the populations of natural enemies by preserving the existing populations in the orchard vicinity or by releasing biocontrol agents. In contrast, our knowledge on the bottom-up regulation processes (i.e. controlled by the host plant) is rather poor.

Genetic resistance to aphids exists in a large number of plant species. In peach, several key genes and quantitative resistances have been detected and are used in breeding programs ${ }^{6}$; however, despite various initiatives taken to increase breeding efficiency, ${ }^{7}$ no resistant cultivar has been released so far, and the durability of these resistances remains uncertain. Plant defense includes physical aspects, such as phloem obstruction by callose deposition or lignification of the cell walls, ${ }^{8}$ and chemical aspects, such as the production of repellent, deterrent or toxic compounds. Nonetheless, the level of susceptibility of the tree is not only determined by the genes involved in the regulation of plant defenses but has also been related to several plant traits associated with shoot growth, size and organization ${ }^{3}$. Shoot composition also plays a role: plant susceptibility was found to vary with the shoot nitrogen $(\mathrm{N}),{ }^{9}$ amino acid $^{10}$ or polyphenol contents, ${ }^{11}$ and with the plant water status. ${ }^{12}$ Tree susceptibility is thus determined by developmental and trophic aspects, i.e. by the balance between plant development (or the emergence of new organs), growth (or size increase of the existing organs) and composition, which could likely be manipulated by cultural practices. Nonetheless, their effects strongly depend on the plant condition (size, shape and 
composition) when the cultural practices are applied. They could therefore produce contradictory results when applied to plants having contrasting life backgrounds. So, water stress was said to affect aphid performances either positively, ${ }^{13}$ negatively ${ }^{14}$ or without any differentiated effect. ${ }^{15}$ Favorable and unfavorable plant traits have probably been affected simultaneously and with different intensities in each experiment, thus leading to opposite conclusions. In order to overcome this difficulty, the plant key variables for susceptibility are to be defined prior to their possible optimization combining various adapted cultural practices.

This study was a first step to link aphid performances to host plant conditions, through focusing on the dualistic interactions between the peach tree and the green peach aphid, and aiming to relate aphid performances to host plant conditions. Young peach trees were submitted to contrasting treatments to increase their phenotypical variability. The trees were then artificially infested with green peach aphids. The effects of those treatments on the trees and the aphids have been described in Rousselin et al.. ${ }^{16}$ In this study, trees were separated in to groups according to the infestation level (high and low infestation), regardless of the treatment they received. The two groups of plants were then compared for: (i) their shoot growth, analyzed from an architectural point of view; (ii) their apex composition in C, N, and phenolic compounds; and (iii) their water status. This large screening, carried out on contrasting culture conditions, ultimately allowed the identification of plant variables related to aphid infestation. It concludes with discussing the robustness of the results and considering their possible extension to different peach genotypes.

\section{Material and Methods}




\subsection{Experimental design}

This study was carried out in Avignon. In February 2012, 80 one-year-old peach trees (cv. Magique, a mid-early white nectarine, susceptible to aphids, grafted on Avimag-Cadaman rootstocks) were planted in $30 \mathrm{~L}$ pots containing a 50\% mix of pozzolan and peat. Being vigorous, the chosen genotype is able to respond rapidly to any fertilization and irrigation treatment, adapting its development or/and its composition. "Magique” is also an interesting cultivar since it is currently widely used in breeding programs, namely for the production of flat peaches due to its resistance to Xanthomonas abricola and its capacity to produce large and sweet fruits. The trees were ca. $1.30 \mathrm{~m}( \pm 0.1 \mathrm{~m})$ tall. The trees were grown outside, well irrigated and fertilized. Between September 10 and November 2, 2012, a preconditioning N treatment, corresponding to mean supplies of 29 and $43 \mathrm{~g} \mathrm{~N}$ tree ${ }^{-1}$, aimed to differentiate two groups of trees by their $\mathrm{N}$ concentration. These concentrations were calculated using the dry weights and $\mathrm{N}$ contents of four trees per group sampled on December 11, 2012. The results were: $1.15 \pm 0.10 \% \mathrm{~N}$ DW (dry weight) and $1.40 \pm 0.14 \% \mathrm{~N}$ DW, respectively.

In January 2013, 48 trees were hand-pruned and settled under an insect-proof shelter. The $\mathrm{N}$ fertilization treatment was carried on in the spring. Between April 25 and June 26, 2013, each group received either 10 applications of a calcium nitrate solution for an overall quantity of $22.2 \mathrm{~g} \mathrm{~N}$ plant $^{-1}$, or two applications (i.e. on June 5 and June 19, 2013) for an overall quantity of $2 \mathrm{~g} \mathrm{~N}$ plant $^{-1}$. From May 3, 2013, the $\mathrm{N}$ treatment was combined with an irrigation treatment: 50\% of each tree group were irrigated daily until drainage occurred and the remaining $50 \%$ of trees received half the amount supplied to the well-irrigated group. Water was provided by two drippers per pot, each with a delivery rate of $2 \mathrm{~L} \mathrm{~h}^{-1}$. Twice a 
week, the irrigation frequency and duration were adjusted to the evaporative demand and plant growth. Prior to infestation, a final tree selection was decided on, in order to reduce the intra group tree variability; nine trees were selected to receive high $\mathrm{N}$ levels with a low water supply; 10 trees were selected to receive both high $\mathrm{N}$ and water supplies; a further 10 were chosen to be restricted in both water and $\mathrm{N}$ levels; and finally, another 12 trees were selected to be restricted in $\mathrm{N}$ supplies, but well-irrigated.

\subsection{Aphid rearing and infestation}

The $M$. persicae aphid colony was established from a single clone (Mp06) of a female collected on a peach tree in March 2013 in Avignon. Aphids were reared in the laboratory on the susceptible peach cultivar GF305, under parthenogenesis-inducing conditions $\left(20 \pm 1^{\circ} \mathrm{C}\right.$, $60-70 \%$ of relative humidity and a photoperiod of 16L:8D). On May 2, 2013, five wingless adult females taken from a 48h cohort were placed on two to three shoots per tree (depending on shoot homogeneity). Aphids were then free to disperse all over the plant.

\subsection{In situ measurements}

Between April 29 and July 9, 2013, measurements were taken once a week on between six and nine shoots per tree: the infested ones and their two closest neighbors of a similar number of leaves. Those 288 shoots were referred to as the parent axes. Their numbers of expanded leaves, corresponding to the number of fully developed growth units, or $\mathrm{GU},{ }^{17}$ were counted and the burst and further growth of their axillary buds was monitored. Each secondary structure was also positioned along the parent axis by the rank of its father GU. Indeed, a 
vegetative axillary bud can either stay dormant or give rise to a short or long ramification (i.e. a rosette or a daughter axis). A bud was referred to as developing bud, between its burst and the full expansion of the rosette's leaves. On July 9, 2013, the basal diameter and stem length (from stem base to the last expanded leaf) were measured on the parent axes.

The plant water status was assessed through the measurement of the minimal water potential using a Scholander pressure bomb, on four trees per treatment modality on May 14, 25 and on 30, and June 7, 13 and 26.

Aphid abundances were estimated once a week, by assigning to each selected shoot one class of infestation as follows: C0 (no aphid), C1 (1 to 5 individuals), C2 (6 to 25), C3 (26 to 125$)$, C4 (125 to 625 ) and C5 (more than 625) accordingly to Grechi et al.. ${ }^{3}$ Estimations were concomitantly made at tree level, counting the number of shoots belonging to each infestation class. The tree infestation index (IF) could then be calculated as described by Grechi et al. ${ }^{3}$ :

$$
\mathrm{IF}=\frac{\sum_{\mathrm{d}=0}^{5} \quad\left(\mathrm{~d} \times \mathrm{f}_{\mathrm{d}}\right)}{5 \times \sum_{\mathrm{d}=0}^{5} \mathrm{f}_{\mathrm{d}}}
$$

where $\mathrm{d}$ is the degree of infestation, i.e. the integer which defined the class of infestation, and $f_{d}$ the frequency of shoots with degree of infestation d, i.e. assigned to class Cd.

\subsection{Biochemical determinations}

For each tree, three non-infested growing apices comprising the terminal meristem and the smallest unfolded leaves (those still standing vertically) were harvested on May 7 and June 5, 2013. Each tree sample represented respectively 8\% (3/38) and 5\% (3/61) of the total number 
of growing parent and daughter axes on May 7 and June 5, 2013, and was thereby considered to be representative of a "tree mean apex" (hereafter abbreviated as tree apex). The samples were immediately immerged in liquid nitrogen, stored at $-80^{\circ} \mathrm{C}$ before being freeze-dried, and then ground in ball mills (MM301, Retsch, Germany) cooled with liquid $\mathrm{N}_{2}$. The total $\mathrm{N}$ concentration was measured using an elemental analyzer (Flash EA 1112, Thermo Finnigan Milan, Italy). Starch and soluble sugars were determined using an enzymatic method as described by Gomez et al.. ${ }^{18}$ The amino acids (identified by their three letters code www.fao.org/docrep/004/y2775e/y2775e0e.htm) were extracted from $10 \mathrm{mg}$ subsamples, homogenized with $1 \mathrm{~mL}$ of sulfosalicylic acid at $2 \%$. The supernatant was collected after centrifugation, buffered to $\mathrm{pH} 2.0$ with potassium hydroxide $2 \mathrm{M}$ and filtered. The amino acids were then derivatized using the AccQ-tag Ultra Derivatization Kit (Waters, Milford, MA USA) before separation and determination by HPLC (Alliance2695, Waters Milford USA) using a Cortecs C18 column (Waters, $4.6 \times 150 \mathrm{~mm}$, particle size $2.7 \mu \mathrm{m}$ ) in line with a 2475 multiwavelength fluorescence detector. Polyphenols (listed in Table 1) were extracted from $20 \mathrm{mg}$ subsamples, homogenized with $1.5 \mathrm{~mL}$ 70\% methanol. The supernatants were collected after centrifugation and filtration. The determinations were then performed by HPLC using an Uptisphere HDO column (Interchim, 3×150 mm, particle size $3 \mu \mathrm{m}$ ) in line with a DAD UV-visible detector (Surveyor, Thermo Fisher Scientific San Jose USA). The measurements were made at two wavelengths (280 or $330 \mathrm{~nm}$ ) and eight polyphenols (listed in Table 1) were identified by their standards. Four unidentified significant polyphenols peaks, resembling hydroxycinnamate derivatives, were also quantified using the 5CQA calibration curve. 


\subsection{Thermal time calculation}

The air daily mean $\left(\mathrm{TI}_{\text {mean }}\right)$, minimum $\left(\mathrm{TI}_{\min }\right)$, and maximum $\left(\mathrm{TI}_{\max }\right)$ temperatures in the insect proof shelter, were based on the climatic weather station located near the experiment. Close linear relationships were fitted (significant at 1\%o level) between air temperatures measured outside and in the insect proof shelter (Hobo V2 logger, U23-002, Onset, Bourne USA) during a similar experiment in 2015. These regressions were used to estimate the thermal time, or Degree-days accumulation (DDA), on the 2013 dataset.

DDA was calculated using a sine wave method. The thresholds for horizontal cutoffs were set to $4^{\circ} \mathrm{C}$ and $27.5^{\circ} \mathrm{C} . .^{19}$

The thermal time requests in DDA for increasing aphid abundance by a factor 5 (i.e. for increasing their degree of infestation or "d" by 1) were calculated for each shoot from infestation start to infestation peak. The start of infestation was considered as either May 2, 2013 (for the infested shoots), or on the median date between when aphids were first observed on the shoot and the date of the previous count. The peak corresponded with the date when the degree of infestation (d) recorded between April 29 and July 9, reached its maximum $\left(d_{\max }\right)$.

\subsection{Data analysis}

All analyses were performed using the R software ( $R$ 2.11.0 software, www.r-project.org/).

The shoots and the trees were divided into low (LI) and high (HI) infested groups. A shoot was assigned to the LI group if it hosted less than 125 aphids throughout the 
observation period, meaning that $d_{\max }$ was 3 or less. The shoots hosting more than 125 aphids (i.e. for which $d_{\max }>3$ ) were assigned to the HI group. After their IF, LI and HI trees were separated into group lower and higher than 0.7: this value typically corresponded to a tree hosting between 125 and 625 aphids on all of constitutive shoots. The thresholds between the LI and HI groups also correspond to the start of exponential increase of the aphid populations and to the apparition of significant damage on the shoot apices. All tested variables were structured according to the LI and HI groups (i.e. they were not statistically different) for $\mathrm{d}_{\max }$ varying between the ranges 0 to 3 and 4 to 5 . For the only exception, the shoots were separated according to the maximum d value in the result section.

Permutation tests performed at a 5\% level, were used to evaluate the differences between LI and HI groups: at shoot level, differences in organ numbers, stem lengths and diameters were assessed; and at tree level, differences were assessed in minimal water potential and apex concentrations of NSC, total levels of N, amino acids and polyphenols. Empirical distributions of each variable effect were derived from 2,500 random assignments of the shoots or trees to the LI or HI groups. ${ }^{24}$ Kolmogorov-Smirnov tests were performed to evaluate how far the LI and HI groups differed: (i) in the distributions of their developing buds, rosettes and daughter axes along the parent axes; and (ii) in their apices amino acid, soluble sugars, and phenol profiles.

\section{Results}

\subsection{Shoot infestation dynamics and aphid dissemination within the tree crown}

After infestation, aphids disseminated along and across the shoots. Between May 14 and June 
13, 2013 (i.e. around infestation peak) the infestation index determined at tree level was significantly correlated (at $1 \%$ o level) to the infestation index of the selected shoots. But aphids were not evenly distributed within the crown. Thus, at infestation peak, only $12 \%$ of the trees had all their selected shoots assigned to the same aphid abundance class, and 55\% of the trees were composed of both LI and HI shoots.

Until infestation peak was reached, the mean thermal time requests for the increase of aphid population at shoot level (Fig. 1a) were independent of infestation severity. The number of aphids per shoot increased by a factor 5 after each $120^{\circ} \mathrm{C}$ DDA, as approximated by the regression slope between the degree of infestation (d) and the thermal time. The aphid population developed during a shorter period of time on the LI shoots. The infestation not only started later but also peaked earlier on those shoots compared to the HI ones (Fig. 2). The first population decline was observed one month earlier on the LI shoots than on the HI ones (i.e. May 13 vs June 10). The aphid populations collapsed rapidly on all shoots after mid-June, as a probable consequence of shoot growth decrease and of temperature increase ${ }^{16}$. No more aphids were present on July 2.

\subsection{Infestation severity increased with shoot development and growth rates}

During the increase to the aphid populations, the thermal time requests for organ emergence (leaves, rosettes and daughter axes) differed greatly between the LI and the HI shoots (Fig. 1 b, c, and d). The LI shoots produced an expanded leaf on their parent axis within $60^{\circ} \mathrm{C}$ DDA and a rosette within $323^{\circ} \mathrm{C}$ DDA. For the HI shoots, those delays were reduced to $47^{\circ} \mathrm{C}$ and $92^{\circ} \mathrm{C}$ DDA, respectively. Furthermore, the emission of daughter axes was almost nil for the 
LI shoots, and varied within the HI group. Thus, still according to the regression slopes (Fig. 1d), a daughter axis was produced every $749^{\circ} \mathrm{C}$ DDA on the shoots assigned to class $\mathrm{C} 4$ at infestation peak, but every $230^{\circ} \mathrm{C}$ DDA on those assigned to class C5.

As a result, the size and shape differed significantly between the LI and HI shoots (Table 2) at the end of the observation period, despite having been selected for homogeneity. Indeed, on April 29, the shoots had a mean number of 9.8 ( \pm 0.09: SE or standard error) leaves and no secondary structures (i.e. no developing bud, rosette or daughter axis). On July 9, the LI and HI groups differed by $10 \%$ in the number of expanded leaves inserted on the parent axes, $20 \%$ in the parent axis basal diameter, by $30 \%$ in the mean internode length. The developing buds transformed rapidly into rosettes and, therefore, their numbers remained low (i.e. below $0.60 \pm 0.04$ and $1.91 \pm 0.06$ for the for the LI and HI shoots respectively) and almost constant. On the LI shoots, axillary development usually stopped at rosette stage while among the HI shoots, the number of daughter axes varied by a factor of 4 between those assigned to class C4 and C5 at infestation peak.

\subsection{Infestation severity increased with architecture complexity}

The LI and HI differed, not only in terms of organ production, but also in the distribution patterns of the secondary structures along their parent axes (Fig. 3). Their repartition among the parents varied between the two groups for the developing buds and the daughter axes, but not for the rosettes. Within the HI group, however, there was no evidence of differences between the shoots assigned to class C4 and class C5, at the infestation peak. The distribution of the daughter axes along the parent axes was similar for the two sub-groups, although their 
numbers differed significantly.

On June 18 (i.e. after infestation peak), 60\% of the rosettes were inserted in median positions (between GU11 and GU20), and less than 6\% in the upper position (above GU20). Developing buds and daughter axes were more widely spread along the HI parents than along the LI ones. Indeed, axillary bud break progressed upwards, in line with the parent axes leaf expansion. Therefore, the highest developing bud was inserted, independently of infestation severity, 3.9 GU ( \pm 0.21) below the upper expanded parent axis leaf. After June 4, however, axillary bud break was reactivated in basal positions, especially for the HI shoots, possibly because aphids had destroyed the shoot top meristems. As a result, the HI parents exhibited a higher proportion of developing buds in the lower and higher positions than the LI parents. The same was observed for the daughter axes, likely as a consequence of the small number of daughters developed by the LI parents.

\subsection{Infestation severity increased with the apex concentration of NSC and amino acids}

On May 7 and June 5, the differences between the LI and HI trees in the apex concentrations were greater for amino acids $\left(\mathrm{AA}_{\text {conc }}\right)$ than for the total $\mathrm{N}\left(\mathrm{N}_{\text {conc }}\right)$, since both variables were related by the following polynomial model:

$$
\mathrm{AA}_{\text {conc }}=\mathrm{N}_{\text {conc }}{ }^{1.2903}-1.2443 \times \mathrm{N}_{\text {conc }}-0.2952 \quad \mathrm{R}_{\text {adj }}^{2}=0.45909 * * *
$$

Tree infestation severity was independent of the total $\mathrm{N}$ but rather related to amino acids. Moreover, the amino acid concentrations (Fig. 4) decreased dramatically over time and to a larger extent for the LI trees than for the HI ones (i.e. by $\pm 60 \%$ vs $46 \%$ ). The only exceptions were Lys, Tyr, Leu and Ile, which increased by a mean of 38\% for the HI trees but 
accounted for less than $4 \%$ in the amino acid pool. The concentrations were lower for the LI trees than for the HI ones, with one significant exception: Thr in June. The differences between LI and HI trees were significant for Asn, Pro, Arg, Gln in May, and for all compounds excepted His, Thr and Ala in June.

No significant differences in the amino acid proportions could be evidenced between the LI and HI trees. That meant that the individual contributions of each amino acid to the pool were only slightly affected by seasonality or infestation severity. In May, for instance, the relative contribution to the amino acid pool of Asn, Pro, Arg, Gln, whose concentrations increased significantly with infestation severity, was of $22 \%$ and $27 \%$ for the LI and HI trees, respectively, and this difference was too low to significantly modify the overall distribution.

Contrary to the amino-acids concentrations, NSC concentrations in the apices (Fig. 5) increased over time for all the compounds but sucrose. Again, the variations were greater for the HI trees than for the LI ones: + 26\% vs $19 \%$ for the soluble sugar pool and $+40 \%$ vs $15 \%$ for starch. However, as observed for the amino acids, the concentrations increased with infestation severity. The only exception (starch in May) was not significant. The differences between LI and HI trees were significant for glucose in May and for sorbitol and sucrose in June, but the soluble sugars distributions were affected neither by infestation severity nor by sampling date.

\subsection{Infestation severity decreased with the apex concentration of polyphenols}

The polyphenol concentrations decreased with infestation severity (Fig. 6). In May, the differences between the LI and HI trees were too small to be significant, but the polyphenol 
concentrations increased dramatically (i.e. by 43\%) over time for the LI trees while remaining stable (i.e. varying by less than 3\%) for the HI ones. As a result, the differences between the two groups became significant in June for all the compounds but CAT. As observed for the primary metabolites, the polyphenol distributions were affected neither by infestation severity nor by sampling date. Indeed, the greatest difference was observed for 5CQA, which contributed with $32 \%$ and $26 \%$ to the polyphenol pool of the LI and HI trees in June, respectively. This difference was again too low to significantly modify the overall distribution.

\subsection{Infestation severity was only indirectly related to tree water status}

Water restriction affected aphid performances but the infestation severity could not be related to the minimal water potential. ${ }^{16}$ Therefore, we searched, among the measured variables to know which varied first, with irrigation treatment, thus integrating the effect of ongoing deficit irrigation, and second, with infestation severity. Both conditions were fulfilled only by stem diameters and to a lesser extent, by the number of daughter axes. Thus, the LI shoots receiving low and high water supplies had a diameter of $4.0 \pm 0.13 \mathrm{~mm}$ and of $4.4 \pm 0.16 \mathrm{~mm}$, respectively. These values reached $4.9 \pm 0.14 \mathrm{~mm}$ and $5.2 \pm 0.15 \mathrm{~mm}$ for the HI trees. Daughter axes were present on only four of the 98 LI shoots, which was too small for statistical tests. However, the HI shoots gave rise to $0.7 \pm 0.22$ daughter axes if water stressed, but to $1.4 \pm 0.24$ ones if well-watered. The apex composition was not affected by the irrigation treatment (results not shown), meaning that the trees adapted to a water restriction concomitantly and proportionally limiting their metabolism and shoot development. 


\section{Discussion}

\subsection{Back to the methodology}

In this first step to better explore the cause-and-effect of plant susceptibility, we chose to work only on one plant genotype whose phenotypical variability was maximized by fertigation treatments. These results will need to be adapted to cultivars differing in their resistance to aphids or drought.

The trees and shoots were sorted by infestation severity, rather than by experimental treatment. At tree level, neither of the classifications matched perfectly and each treatment comprised various proportions of LI and $\mathrm{HI}$ trees. About $90 \%$ of the well-fertilized trees were highly infested whatever the irrigation treatment; while respectively $55 \%$ and $45 \%$ of the low $\mathrm{N}$ ones were assigned to the HI group depending on whether or not they had been well watered. Furthermore, most trees were composed of shoots of different infestation levels. Nevertheless, low shoot infestation could not be associated with a late infestation start which left the aphid colonies with insufficient time to fully develop. Thus, infestation peak was usually met earlier on the LI shoots than on the HI ones, which confirmed the difference of suitability for aphids between the two shoot groups.

Aphid performances were linked to organ development at shoot level and to organ composition only at tree level. Indeed, monitoring growth from an architectural point of view was not feasible on whole trees, so the focus was, therefore, put on the individual shoots. On the contrary, apex composition could not be determined at shoot level, since infestation damaged the apices (drying-up, deposition of honeydew), being therefore assessed by a 
collection of approximately 5\% of the tree growing apices. The samples were large enough to represent a pertinent marker of the "plant biochemical attractiveness" to aphid, and were composed of apices which were not infested because of their young age and/or position.

The biochemical determinations were performed on growing apices rather than on phloem sap. Indeed, phloem composition, velocity and volume flow were related to the instant aphid intake, but varied greatly over day ${ }^{20}$ and with plant phenology. ${ }^{21}$ That could explain why infestation severity could hardly be related to markers of instant plant functioning (i.e. water potential and leaf-air exchanges). ${ }^{16}$ The mean apex concentrations were more stable and resume the prevailing conditions during apex formation to which the aphids were submitted. Indeed, the leaves act as sinks for all nutrients, including carbohydrates, ${ }^{22}$ until their full expansion.

\subsection{Plant susceptibility to aphids as related to its functional balance}

Aphid performances appeared to be related first, to shoot development (i.e. leaf formation and secondary ramification); secondly to shoot growth (i.e. stem length and diameter); and thirdly, to apex concentrations in amino acids, NSC and polyphenols. Different aspects of plant functional equilibrium could affect the insect performances with possible antagonistic effects. Moreover, if and how each of the cited variables affected plant susceptibility to aphids directly or varied as a side effect of the functional dependence between organ formation, growth, and composition, remains to be discussed.

\subsection{Main determinants of plant susceptibility}


Firstly, shoot development and growth plays an active role in plant susceptibility to aphids. Indeed, aphids hosting on shoot apices or young leaves prefer long shoots (i.e. those having the highest growth rate), ${ }^{9}$ since they received the highest amounts of water and nutrients. ${ }^{25}$ Our results confirmed these observations, as the HI and LI shoots differed in the size (length and diameter) of their parent axes, and in their ramification patterns. The HI shoots also had the highest conductive capacities as attested to their higher diameters. ${ }^{26}$ Indeed, due to the relatively small size of our trees, the aphids leaving their hosting shoot were able to roam within the whole crown to find another favorable feeding site, potentially a further HI shoot.

Secondly, aphids are affected by shoot and/or phloem composition. Thus, in choice tests, aphids prefer the excised leaves sampled on well-fertilized plants. ${ }^{27}$ This preference has been related to (i) higher amino acids and soluble sugars concentrations, considered as markers of food quality, ${ }^{27}$ (ii) more favorable N/C ratio of the phloem sap, and (iii) lower contents in detrimental nutrients, among which phenolic compounds. ${ }^{28}$ Sap viscosity also increased exponentially with phloem concentration, ${ }^{12}$ which may be osmotically stressful for the phloem feeders. ${ }^{27}$

(i) Food quality is related to higher concentrations of several specific amino acids (Thr, His, Phe, Val, Lys, Leu, Ile, Met and Trp), but only present in small amounts in a plant. Therefore, they need to be synthetized by the obligatory endosymbiont (Buchnera aphidicola) of M. persicae, which uses Glu or Gln and sucrose as N and C sources. ${ }^{29}$ This may explain why $43 \%$ of the variation, in the intrinsic rate of population increase, is related to the amino acids concentrations of Tyr, Ala, Leu, and Glu. ${ }^{30}$ In our study, the LI and HI trees differed significantly with regard to their concentration at infestation peak of Tyr, Ala, Leu, and Glu 
taken together, which was 0.13 and $0.20 \%$ DW, respectively. As the composition of the pool was affected neither by infestation severity nor by seasonality, this difference was a consequence of the higher amino acid content of the HI trees in June. The essential amino acids represented approximatively $15 \%$ of the pool, while Tyr, Ala, Leu, and Glu taken together accounted for one third.

(ii) The plant aphid interactions also depend on sucrose concentrations, as they lead to large variations in the N/C ratio. Since aphids feed passively, they have to excrete the $C$ assimilated in excess. ${ }^{10}$ On artificial diets correctly supplemented in essential amino acids, aphid performances decrease when sucrose concentrations are above $1 \mathrm{M}$ and the threshold value for survival is $1.5 \mathrm{M}^{31}$ Aphid nutritional requirements could also be resumed by the phloem amino acid/soluble carbohydrate ratio, whose optimum value for insect welfare varies between $0.1(\mathrm{w} / \mathrm{w})$ and $0.2 .^{10,27}$ In our study, the LI and HI trees differed significantly in their concentration of glucose in May, and sucrose and sorbitol in June. These forms were involved in C transport, respectively during and after store mobilization (see infra), and were therefore likely to be present in large amounts in the phloem. Their concentrations were always higher in the $\mathrm{HI}$ trees than in the LI ones, which was more than compensated by the greater differences regarding amino acid concentrations. Indeed, the soluble sugars/amino acids ratios of the apices were lower for the HI trees than for the LI ones in May (6.9 vs 8.3) and June (16.4 vs 24.8). Whether or not this conclusion was applicable to the phloem sap remained nonetheless questionable.

(iii) The nutritional quality of the host plant could be altered by the presence of defense compounds, which include secondary compounds such as alkaloids, glucosinolates, 
terpenoids and phenolics. Among the phenolics, flavonoids, including condensed tannins and phenolic acids, ${ }^{11,28}$ have been identified as defense compounds. Nevertheless, phenols taken individually or cumulatively are poor predictors of plant susceptibility due to the existence of antagonistic or synergistic effects with other compounds like jasmonic and salicylic acids. ${ }^{28}$ In the present study, most of the identified polyphenols act as defense compounds on peach or against green peach aphids. ${ }^{11}$ Their concentrations remained stable over time for the HI trees but increased dramatically in the non-infested shoots of the LI trees.

\subsection{Functional dependence between organ development, growth and composition}

Organ development and composition are strongly entangled. Indeed, the faster development and growth observed in the HI group could be related to their higher nutrient availability compared to the LI trees. Thus, in May, the concentrations were significantly higher in the HI trees for only four amino acids, all of them being involved in $\mathrm{N}$ transport and mainly issued from store remobilization. Indeed, Arg and Asn are long identified as major reserve compounds. ${ }^{32}$ The transport of the remobilized $\mathrm{N}$ towards the growing shoots occurred mainly with Asn, Gln and Glu. ${ }^{21}$ Proline (Pro) was also involved in store management, even though it is mainly known as an osmoprotectant of plant cells against drought. In regard to the $\mathrm{C}$ stores, glucose-which was the only sugar whose concentration increased significantly with infestation severity in May—is the main product of starch hydrolysis.

In June, the concentrations of almost all amino acids varied significantly between the LI and HI trees, indicating an overall higher metabolic and transport activity of the HI trees. Indeed, most of the overage $\mathrm{N}$ is mainly stored in the leaves, where it is present in the form of 
Rubisco. This hypothesis was also coherent with the variations observed for NSC, since sucrose and sorbitol are the main primary photosynthetic products exported by mature peach leaves to the growing sinks. ${ }^{33}$ Furthermore, there is a consensus stating that phenolic synthesis is favored when growth is limited by poor $\mathrm{N}$ supply, thus increasing the amounts of $\mathrm{C}$ that are able to be mobilized in the defense metabolism. ${ }^{28}$ The low polyphenols concentrations in the HI trees could, therefore, be considered as suitable markers of their high-growth potential.

Shoot growth is adapted to $\mathrm{N}$ availability, which affects the shoot elongation rate, ${ }^{23}$ the secondary ramification, ${ }^{24}$ and, in turn, infestation severity. However, infestation severity increased with leaf $\mathrm{N}$ content, but only upon a threshold value, Sauge et al. ${ }^{9}$ which could correspond to the instance where $\mathrm{N}$ was not limiting growth. In our experiment, leaf $\mathrm{N}$ was kept below that threshold. ${ }^{16}$

Tree water deficit is known to uncouple growth from photosynthesis and, thus, imbalances the source sink relationship. As a consequence of growth cessation, the shoots concentrations increased in soluble sugars and in amino acids no longer involved in protein synthesis. ${ }^{34}$ Osmoprotectants such as Pro, flavonoids or glucosinolates, which could also be active in plant resistance to pests, ${ }^{35}$ were concomitantly accumulated. In our study, however, the irrigation treatment did not affect organ composition, but reduced the stem diameters and the number of daughter axes, which are both markers of infestation severity. The lower stem diameters of the LI shoots when compared to the HI ones, could be a consequence of the lower leaf area (i.e. transpiring surface) of those shoots, or related to a shortage of xylem fluxes by insufficient water supply, which in turn reduced the diameter of the vessels. ${ }^{26}$ This could explain why the water availability modified the link between shoot growth and aphid 
abundance. $^{16}$

\section{Conclusion}

Peach susceptibility to aphids encompasses simultaneously trophic and developmental aspects. However, in the range of variation imposed by our treatments, the differences in the shoot apices composition which led to contrasting infestation severities, could be seen as markers of shoot vigor.

Tree suitability to aphids could, therefore, be lowered using various practices to diminish the vigor of the trees and shoots. Possible practices include (i) low pruning intensity, which decreases the individual shoot growth by increasing their number, (ii) deficit irrigation and (iii) low $\mathrm{N}$ supply. These practices, however, need to be applied for long periods of time (several years), as their final outcomes varied greatly according to the initial condition of the trees. Their intensity should also be adapted to the cultivars and environmental conditions.

\section{Acknowledgements}

This work was funded by the ARIMNET (ANR-12-AGR-0001): 'APMed’ project (Apple and Peach in Mediterranean orchards) and by the 'RegPuc' project (Quelles stratégies d'irrigation et de fertilisation pour réguler les populations de pucerons verts en vergers de pêcher). The RegPuc project is led by the Ministry of Agriculture and Food and the Ministry for an Ecological and Solidary Transition, with the financial support of the French Biodiversity Agency on Resistance and Pesticides research call, with the fees for diffuse pollution coming from the Ecophyto plan. We would like to thank M. Couëllier, V. Serra, J. P. Lacroze and F. 
Bouvery for their technical help and their contribution to the field measurements; S. Serino,

D. Bancel, P. Laugier and E. Rubio for performing the chemical analyses; M-N. Corre for their technical advice and J-L. Poëssel for their careful proofreading.

\section{References}

1. Bass C, Puinean AM, Zimmer CT, Denholm I, Field LM, Foster SP, Gutbrod O, Nauen R, Slater R and Williamson MS, The evolution of insecticide resistance in the peach potato aphid, Myzus persicae. Insect Biochem Mol Biol 51:41-51 (2014).

2. Bevacqua D, Grechi I, Genard M and Lescourret F, The consequences of aphid infestation on fruit production become evident in a multi-year perspective: Insights from a virtual experiment. Ecol Model 338:11-16 (2016).

3. Grechi I, Sauge MH, Sauphanor B, Hilgert N, Senoussi R and Lescourret F, How does winter pruning affect peach tree - Myzus persicae interactions? Entomol Exp Appl 128:369-379 (2008).

4. Manachini B, Casati P, Cinanni L and Bianco P, Role of Myzus persicae (Hemiptera: Aphididae) and its secondary hosts in Plum Pox Virus propagation. J Econ Entomol 100:1047-1052 (2007).

5. Rousselin A, Bevacqua D, Sauge MH, Lescourret F, Mody K and Jordan MO, Harnessing the aphid life cycle to reduce insecticide reliance in apple and peach orchards. A review. Agron Sustain Dev 37:38 (2017).

6. Sauge MH, Lambert $\mathrm{P}$ and Pascal T, Co-localisation of host plant resistance QTLs affecting the performance and feeding behaviour of the aphid Myzus persicae in the peach 
tree. Heredity 108:292-301 (2012).

7. Laurens F, Aranzana MJ, Arus P, Bassi D, Bink M, Bonany J, Caprera A, CorelliGrappadelli L, Costes E, Durel CE, Mauroux JB, Muranty H, Nazzicari N, Pascal T, Patocchi A, Peil A, Quilot-Turion B, Rossini L, Stella A, Troggio M, Velasco R and van de Weg E, An integrated approach for increasing breeding efficiency in apple and peach in Europe. Hortic Res 5:11 (2018).

8. Sun M, Voorrips RE, Steenhuis-Broers G, van't Westende W and Vosman B, Reduced phloem uptake of Myzus persicae on an aphid resistant pepper accession. BMC Plant Biol 18:138 (2018).

9. Sauge MH, Grechi I and Poëssel JL, Nitrogen fertilization effects on Myzus persicae aphid dynamics on peach: vegetative growth allocation or chemical defence? Entomol Exp Appl 136:123-133 (2010).

10. Ryan GD, Shukla K, Rasmussen S, Shelp BJ and Newman JA, Phloem phytochemistry and aphid responses to elevated $\mathrm{CO}_{2}$, nitrogen fertilization and endophyte infection. Agric Forest Entomol 16:273-283 (2014).

11. Ranger CM, Singh AP, Frantz JM, Cañas L, Locke JC, Reding ME and Vorsa N, Influence of Silicon on Resistance of Zinnia elegans to Myzus persicae (Hemiptera: Aphididae). Environ Entomol 38:129-136 (2009).

12. Will T, Hewer A and van Bel AJE, A novel perfusion system shows that aphid feeding behaviour is altered by decrease of sieve-tube pressure. Entomol Exp Appl 127:237-245 (2008).

13. Oswald CJ and Brewer MJ, Aphid-barley interactions mediated by water stress and barley 
resistance to Russian wheat aphid (Homoptera: Aphididae). Environ Entomol 26:591-602 (1997).

14. Hale BK, Bale JS, Pritchard J, Masters GJ and Brown VK, Effects of host plant drought stress on the performance of the bird cherry-oat aphid, Rhopalosiphum padi (L.): a mechanistic analysis. Ecol Entomol 28:666-677 (2003).

15. Pons X and Tatchell GM, Drought stress and cereal aphid performance. Ann Appl Biol 126:19-31 (1995).

16. Rousselin A, Sauge MH, Jordan MO, Vercambre G, Lescourret F and Bevacqua D, Nitrogen and water supplies affect peach tree-green peach aphid interactions: the key role played by vegetative growth. Agric Forest Entomol 18:367-375 (2016).

17. Kervella J, Pages L and Genard M, Growth context and fate of axillary meristems of young peach trees. Influence of parent shoot growth characteristics and of emergence date. Ann Bot 76:559-567 (1995).

18. Gomez L, Bancel D, Rubio E and Vercambre G, The microplate reader: an efficient tool for the separate enzymatic analysis of sugars in plant tissues - validation of a micromethod. J Sci Food Agric 87:1893-1905 (2007).

19. Higley LGH, Pedigoand LP and Ostlie KR, DEGDAY: A Program for Calculating Degree-days., and Assumptions Behind the Degree-day Approach. Environ Entomol 15:999-1016 (1986).

20. Peuke AD, Rokitta M, Zimmermann U, Schreiber L and Haase A, Simultaneous measurement of water flow velocity and solute transport in xylem and phloem of adult plants of Ricinus communis over a daily time course by nuclear magnetic resonance 
spectrometry. Plant Cell Environ 24:491-503 (2001).

21. Malaguti D, Millard P, Wendler R, Hepburn A and Tagliavini M, Translocation of amino acids in the xylem of apple (Malus domestica Borkh.) trees in spring as a consequence of both N remobilization and root uptake. J Exp Bot 52:1665-1671 (2001).

22. Bieleski RL and Redgwell RJ, Sorbitol versus sucrose as photosynthesis and translocation products in developing apricot leaves. Aust J Plant Physiol 12:657-668 (1985).

23. Jordan MO, Vercambre G, Gomez L and Pages L, The early spring N uptake of young peach trees (Prunus persica) is affected by past and current fertilizations and levels of C and N stores. Tree Physiol 34:61-72 (2014).

24. Jordan MO, Wendler R and Millard P, The effect of autumn N supply on the architecture of young peach (Prunus persica L.) trees. Trees-Struct Funct 23:235-245 (2009).

25. Larson KC and Whitham TG, Competition between gall aphids and natural plant sinks: Plant architecture affects resistance to galling. Oecologia 109:575-582 (1997).

26. Bolat I, Dikilitas M, Ercisli S, Ikinci A and Tonkaz T, The Effect of Water Stress on Some Morphological, Physiological, and Biochemical Characteristics and Bud Success on Apple and Quince Rootstocks. Sci World J 2014:769732 (2014).

27. Nowak $\mathrm{H}$ and Komor E, How aphids decide what is good for them: experiments to test aphid feeding behaviour on Tanacetum vulgare (L.) using different nitrogen regimes. Oecologia 163:973-984 (2010).

28. Mutikainen P, Walls M, Ovaska J, Keinanen M, Julkunen-Tiitto R and Vapaavuori E, Herbivore resistance in Betula pendula: Effect of fertilization, defoliation, and plant genotype. Ecology 81:49-65 (2000). 
29. Sandstrom J and Moran N, How nutritionally imbalanced is phloem sap for aphids? Entomol Exp Appl 91:203-210 (1999).

30. Cole RA, The relative importance of glucosinolates and amino acids to the development of two aphid pests Brevicoryne brassicae and Myzus persicae on wild and cultivated brassica species. Entomol Exp Appl 85:121-133 (1997)

31. Douglas AE, Price DRG, Minto LB, Jones E, Pescod KV, Francois CLMJ, Pritchard J and Boonham N, Sweet problems: insect traits defining the limits to dietary sugar utilisation by the pea aphid, Acyrthosiphon pisum. J Exp Biol 209:1395-1403 (2006).

32. Titus JS and Kang SM, Nitrogen metabolism, translocation, and recycling in apple trees. Hortic. Rev. 4: 204-246 (1982).

33. Moing A, Carbonne F, Rashad MH and Gaudillere JP, Carbon fluxes in mature peach leaves. Plant Physiol 100:1878-1884 (1992).

34. Jimenez S, Dridi J, Gutierrez D, Moret D, Irigoyen JJ, Moreno MA and Gogorcena Y, Physiological, biochemical and molecular responses in four Prunus rootstocks submitted to drought stress. Tree Physiol 33:1061-1075 (2013).

35. Nachappa P, Culkin CT, Saya PM, Han JL and Nalam VJ, Water Stress Modulates Soybean Aphid Performance, Feeding Behavior, and Virus Transmission in Soybean. Front Plant Sci 7:552 (2016). 


\section{Figures Captions}

Fig 1. Aphid and shoot development between infestation start and infestation peak as a function of Degree Days Accumulation (DDA). The calculations were done separately for each shoot, considering only the dates when each shoot was first assigned to an aphid abundance class $\left(C_{d}, 1 \leq d \leq d_{\max }, d_{\max }\right.$ : degree of infestation at infestation peak). (a) represent the DDA (means and standard errors) needed to increase $\mathrm{d}$ by one for $1 \leq \mathrm{d} \leq \mathrm{d}_{\max }$. The concomitant shoot development, i.e. organ production during the period of aphid development, is detailed for the numbers of expanded leaves (b) rosettes (c) and long daughter axes (d). The 98 LI shoots $\left(d_{\max } \leq 3\right)$ are represented by solid blue lines, the $190 \mathrm{HI}$ ones $\left(4 \leq d_{\max } \leq 5\right)$ by solid red lines. In (d), the shoots for which $d_{\max }=4$ (red two-dashed line, $\mathrm{N}=158$ ) are separated from the ones for which $d_{\max }=5$ (red long-dashed line, $\mathrm{N}=32$ ) since the two groups behave differently. Means (ranked in ascending order) were significantly different if coded with different letters.

Fig 2. Proportion (in \%) of shoots at different infestation stages during the infestation period for (a) low infested shoots $(\mathrm{N}=98)$ and $(\mathbf{b})$ high infested shoots $(\mathrm{N}=190)$. The lines represent the proportion of shoots on which infestation has started (solid lines), reached its maximum (long-dashed lines) or declined (dotted lines). Each area thus represents a different infestation stage as indicated in the plots: (i) healthy shoots hosting no aphids; (ii) shoots on which the infestation has started but not reached their peaks, i.e. on which the aphid populations increased; (iii) shoots at infestation peak during which the aphid populations 
remained at their highest levels; and (iv) shoots on which the populations declined.

Fig 3. Repartition along the parent axes growth units (GU) of the developing buds (hatched bars), rosettes (pale gray bars) and daughter axes (black bars) for the 98 LI shoots (a) and the HI ones, separated after the value of $d_{\max }$ : (b) $d_{\max }=4$ for 158 individuals and (c) $d_{\max }=5$ for 32 individuals. The bars stand for the mean numbers of secondary structures raised by each GU, and the lines for the standard errors calculated after the sums of developing buds, rosettes and daughter axes.

Fig 4. Mean amino acid concentrations (in \%o DW) in the apices of 12 LI (blue bars) and 29 HI (red bars) trees. The black lines represent the standard errors. The amino acids whose concentrations differed significantly between the LI and HI trees are identified by asterisk.

Fig 5. Mean non-structural carbohydrate concentrations (in \% DW) in the apices of $12 \mathrm{LI}$ (blue bars) and $29 \mathrm{HI}$ (red bars) trees. The black lines represent the standard errors. The carbohydrates whose concentrations differed significantly between the LI and HI trees are identified by stars.

Fig 6. Mean polyphenol concentrations (in \%o DW) in the apices of 12 LI (blue bars) and 29 HI (red bars) trees. The black lines represent the standard errors. The polyphenols are represented by their abbreviations (see Table 1), and the four undefined (Undef) compounds were numbered after their retention time in HPLC. The polyphenols whose concentrations 
differed significantly between the LI and HI trees are identified by asterisks. 
Table 1. Abbreviation of the polyphenols.

\section{Polyphenol}

Abbreviation

Chlorogenic acid (5-caffeoyl quinic acid) 5CQA

Kaempferol-3-glucoside

K3Glc

Kaempferol-3-galactoside

K3Gal

Quercetin-3-glucoside

Q3Glc

3,5-dicaffeoylquinic acid

3,5-DiCQ

Hyperoside

Q3Gal

Neo-chlorogenic acid (3-caffeoylquinic acid)

3CQA

Catechin

CAT 
Table 2. Final dimensions (means and standard errors) of the selected shoots according to their infestation severity. The measurements were taken on July 9, 2013, for stem lengths and on July 2, 2013, for the number of organs. The mean internode lengths were calculated for each shoot as the ratio between the length and the number of expanded leaves. The $98 \mathrm{LI}$ and 190 HI shoots (among which 158 and 32 were assigned to class C4 and C5 at infestation peak, respectively) differed significantly for all variables.

$\begin{array}{lll}\text { Parameters measured } & \text { LI shoots } & \text { HI shoots }\end{array}$

\begin{tabular}{lcc}
\hline Parent axis length (cm) & $27.0 \pm 1.4$ & $38.4 \pm 1.4$ \\
Parent axis diameter (mm) & $4.2 \pm 0.6$ & $5.1 \pm 0.1$ \\
Expanded leaves (number per parent axis) & $20 \pm 0.35$ & $22 \pm 0.4$ \\
Mean internode length (cm) & $1.3 \pm 0.2$ & $1.7 \pm 0.1$ \\
Developing buds (number per parent axis) & $0.05 \pm 0.02$ & $0.33 \pm 0.05$ \\
Rosettes (number per parent axis) & & $7.0 \pm 0.3$
\end{tabular}

Daughter axes (number per parent axis) $\quad 0.08 \pm 0.05 \quad$ Class C4: $0.75 \pm 0.13$

Class C5: $2.87 \pm 0.69$ 

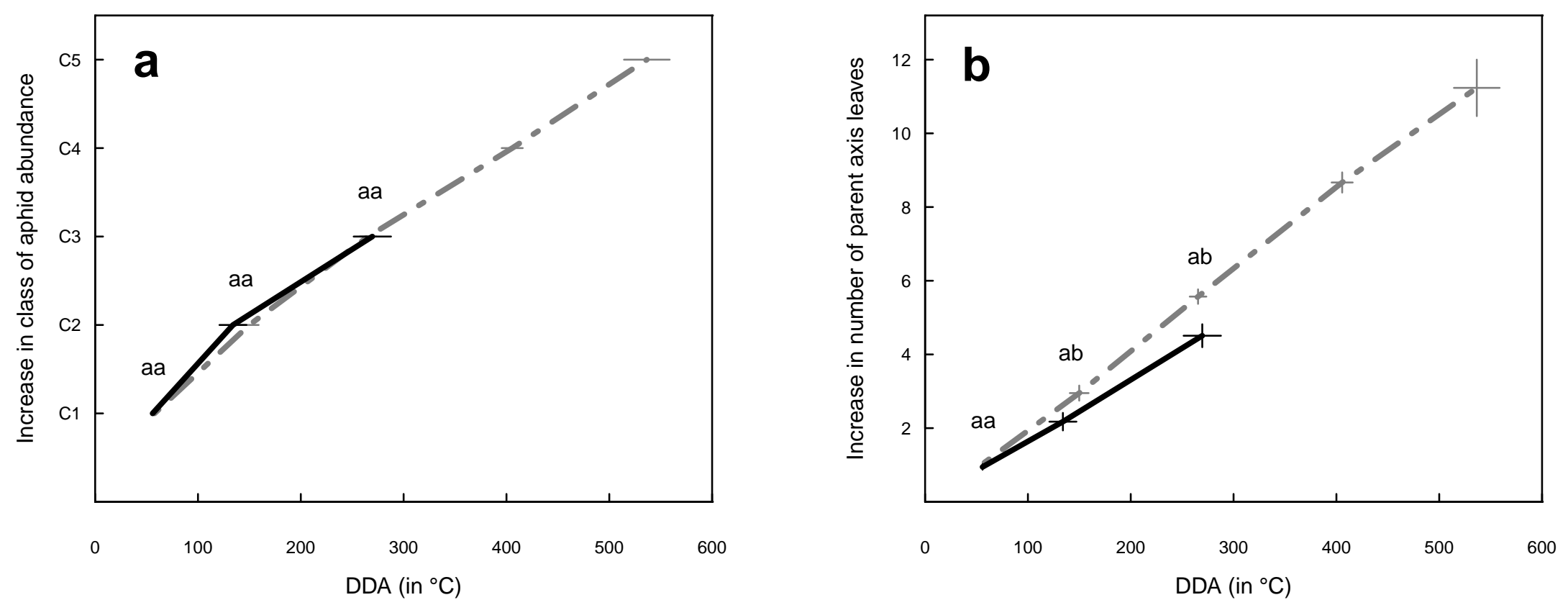

(1)
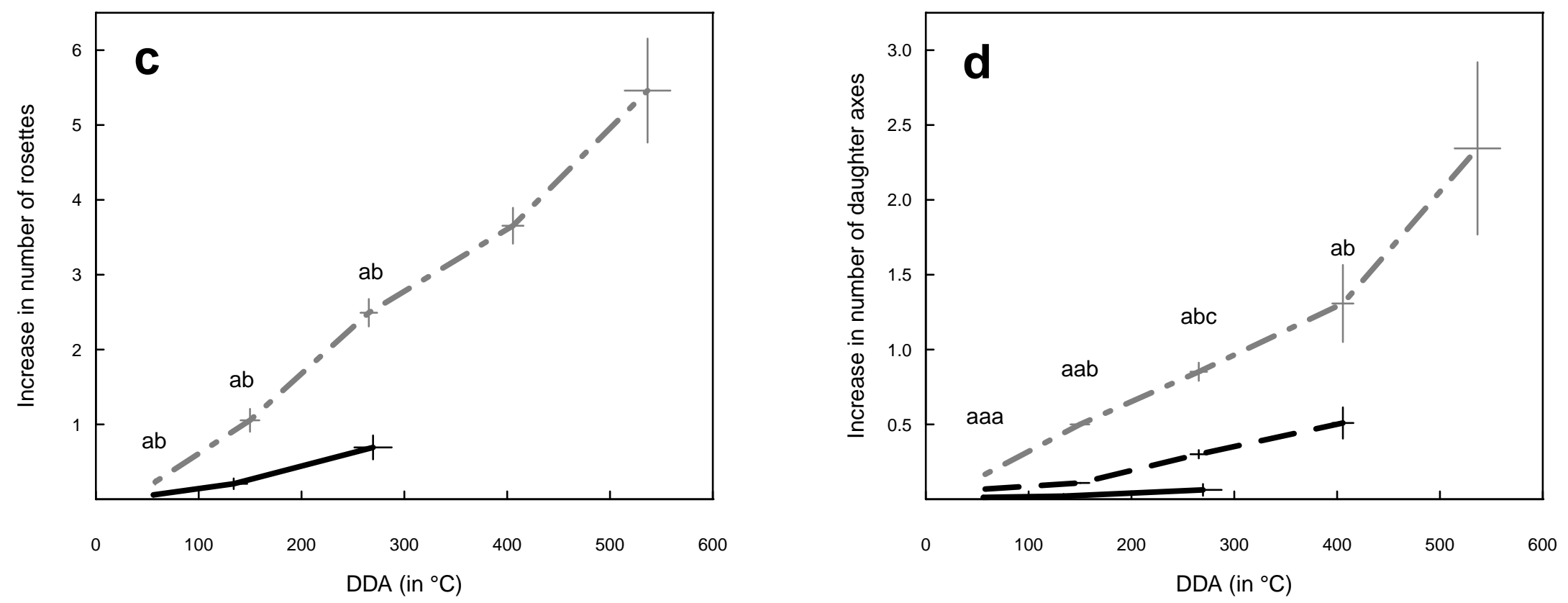

Comment citer ce document: is protected by copyright: All rights reserved. 
(1)
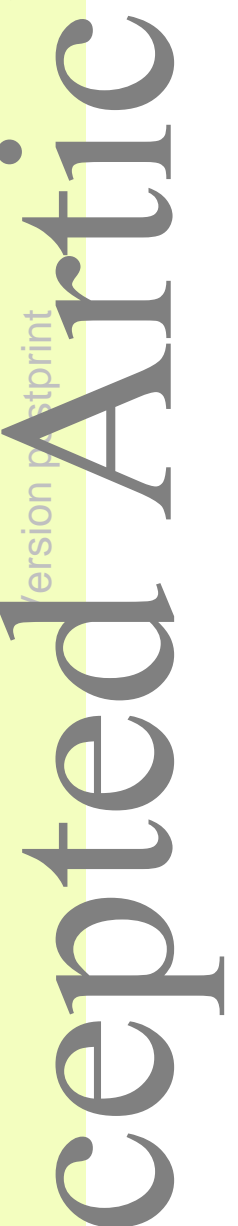

$\circlearrowright$
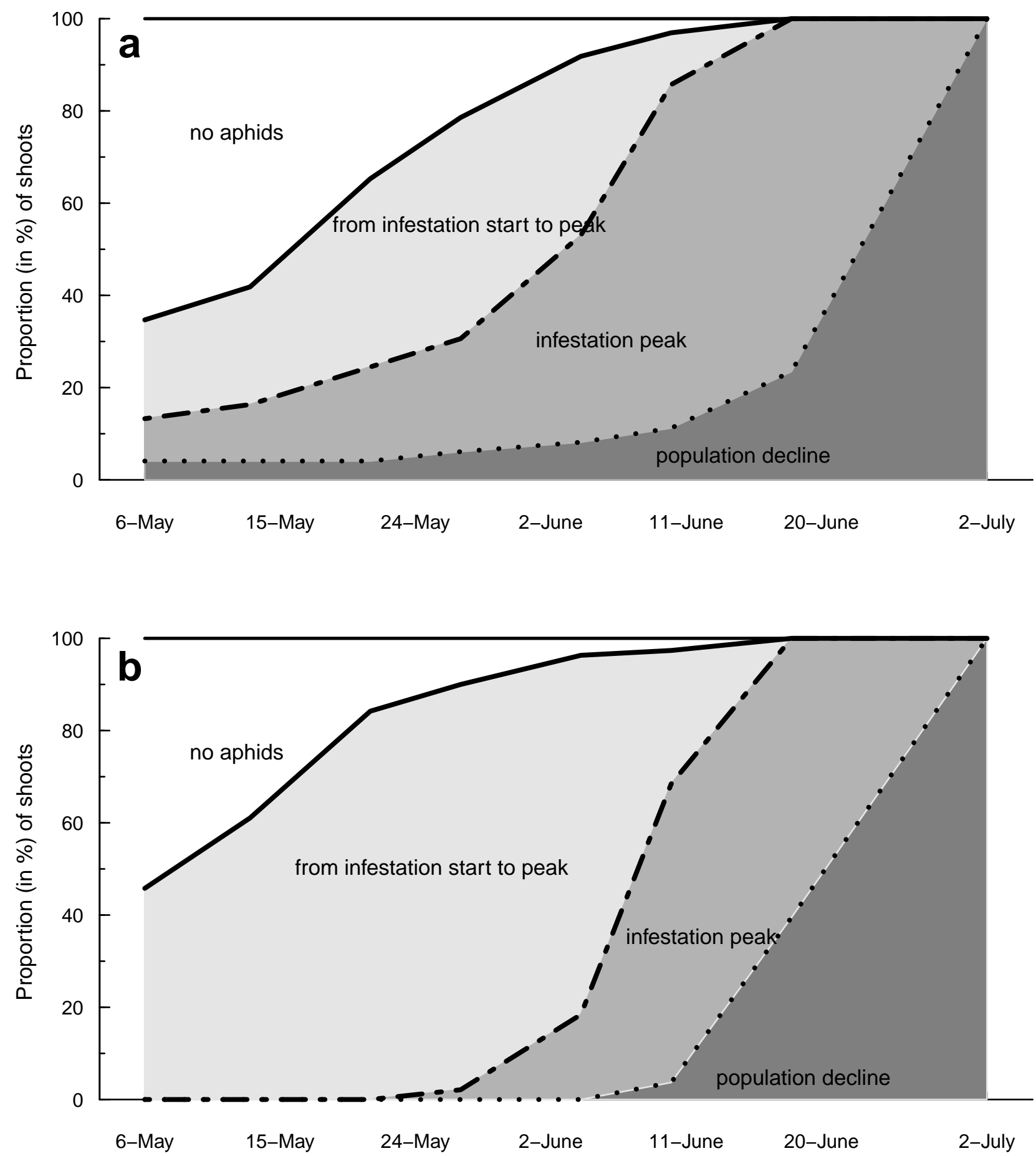

Jordan, M. O. (Auteur de correspondaThis article is protected by copyright. All rights reserved.d
rowth traits of the peach tree may induce higher infestation rates of the green peach aphid, Myzus persicae (Sulzer). Pest Management Science, In Press, Accepted manuscript online: 09 August 


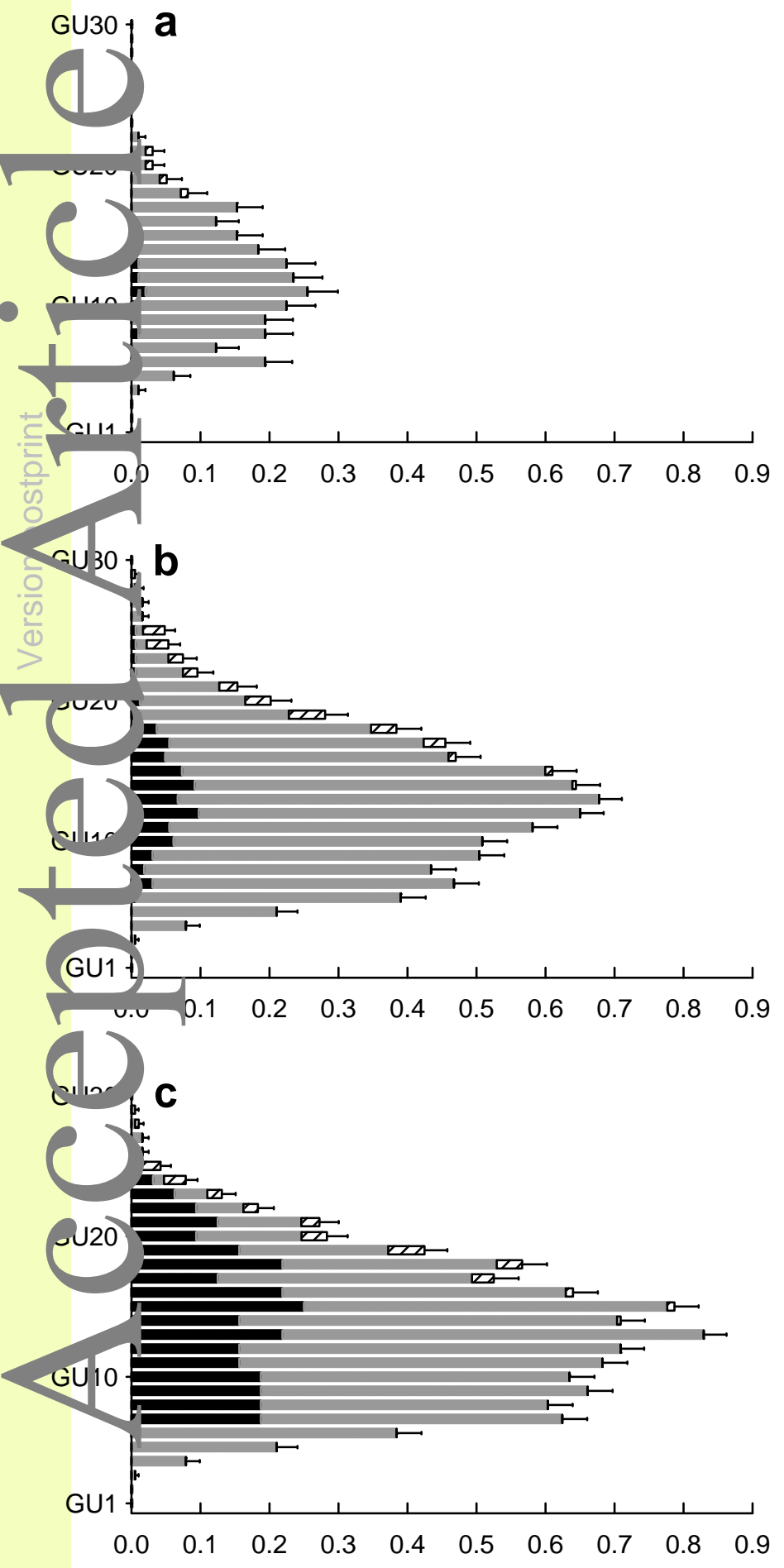

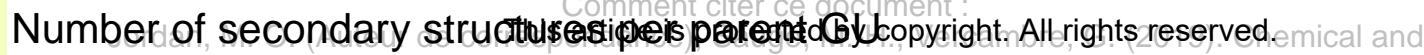
growth traits of the peach tree may induce higher infestation rates of the green peach aphid, Myzus persicae (Sulzer). Pest Management Science, In Press, Accepted manuscript online: 09 August 


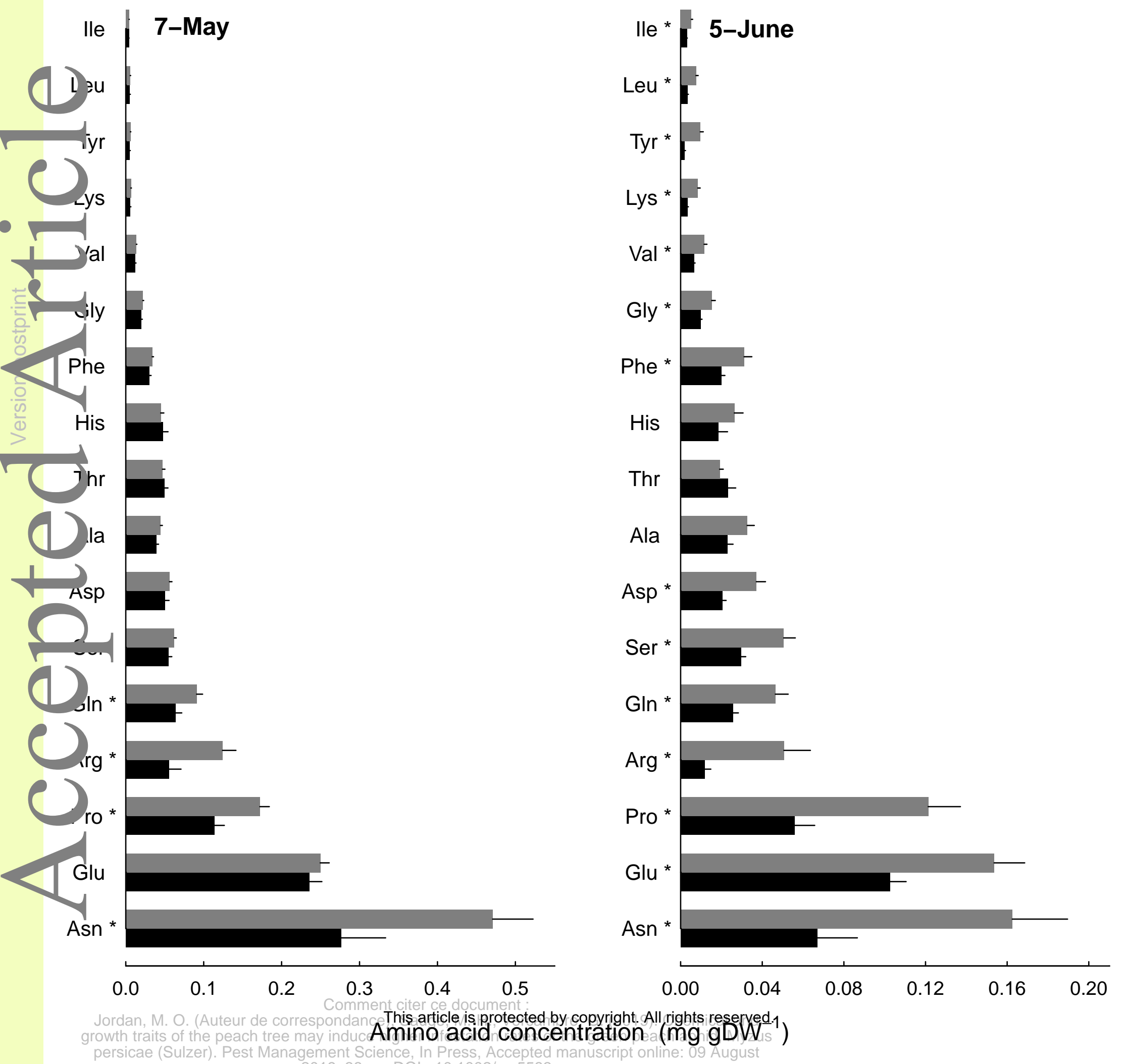


